\title{
The Calculation of the Contribution of Freely Rotating Groups to Electron Scattering by Gases
}

\author{
J. KARLE \\ Chemistry Department, University of Michigan, Ann Arbor, Michigan
}

(Received February 19, 1945)

\begin{abstract}
The contribution from freely rotating a toms to the intensity of electron scattering by gaseous molecules is obtained by means of an asymptotic evaluation of an integral, using the saddle point method. It is possible now to estimate the effect of the approximation in using the Debye formula, which is a specialization of the derived series, and, if desired, to obtain a higher degree of accuracy. Two cases are considered numerically.
\end{abstract}

$I^{1}$ $\mathrm{N}$ many molecules in the gaseous state internal vibrations of large amplitude and even practically unhindered rotation are possible. This, of course, is well known from heat capacity and spectroscopic investigations. In his paper concerning the effect of various types of internal motion in gaseous molecules on the resulting intensity of scattered electrons, P. Debye ${ }^{1}$ made an important contribution to the calculation of the effect of a freely rotating group in a special type of molecule by evaluating the integral

$$
\int \frac{\sin s r}{s r} d W
$$

by means of an asymptotic formula. In the integrand, $s=(4 \pi / \lambda) \sin (\theta / 2), r$ is the varying distance of a rotating atom from another whose position in space is fixed, and which is not on the axis of rotation (for then $r$ would not vary) and $d W$ is the probability of finding the rotating atom in any position on the circle of rotation. $s$ corresponds to $s k$ in Debye's treatment.

It is the purpose of this paper to afford the investigator a means for estimating the effect of

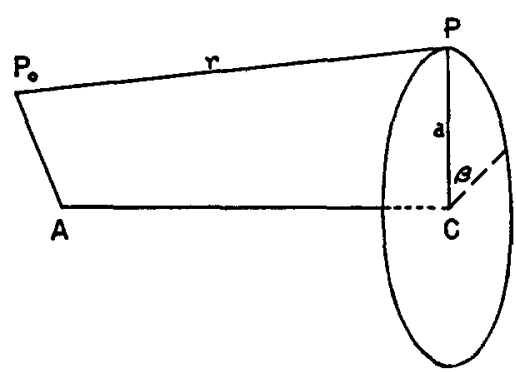

FIG. 1.

${ }^{1}$ P. Debye, J. Chem. Phys. 9, 55 (1941). the approximation introduced by using the Debye formula and for obtaining a greater accuracy, if desired. The general case, in which there are no restrictions on the molecular parameters, is treated and an expression is derived which is the sum of four asymptotic series. It can then be shown that Debye's formula is obtained if one takes the first term in two of the series, neglecting the other two, and specializes it to Debye's example. By means of these semi-convergent series it is easy to estimate the approximation introduced by using the Debye formula (or, more generally, the formula which is the sum of the first terms in two of the series) and, in addition, higher accuracy may be obtained by including additional terms.

\section{MATHEMATICAL DEVELOPMENT}

Consider the point, $P$, rotating with fixed radius of rotation, $a$, about the axis $A C$. Its distance, $r$, from a fixed point in space, $P_{0}$, can be shown to be

where

$$
r=\left(c_{1}-c_{2} \cos \beta\right)^{\frac{1}{2}}
$$

$$
c_{1}=\left(l_{2}{ }^{2}+l_{1}{ }^{2}\right) / 2, \quad c_{2}=\left(l_{2}{ }^{2}-l_{1}{ }^{2}\right) / 2,
$$

and $\beta$ is the angle of rotation. $r_{\max }=l_{2}, r_{\mathrm{min}}=l_{1}$, and, clearly, $\beta=0$ when $r=l_{1}$.

The contribution to the total scattering function from a pair of atoms at $P$ and $P_{0}$, respectively, is given by $(\sin s r) / s r$. It is necessary however to consider the average contribution by the changing distance in the course of a rotation. Thus, we obtain an integral of the form of (1) which is an averaging of the contributions from all positions of orientation. We have by 
expressing $r$ as in (2) above,

$$
I=\int_{0}^{2 \pi} \frac{\sin \left[x(1-p \cos \beta)^{\frac{1}{2}}\right] d \beta}{x(1-p \cos \beta)^{\frac{1}{2}} 2 \pi},
$$

where $x=s c_{1}^{\frac{1}{2}}, p=c_{2} / c_{1}$ and $d \beta / 2 \pi=d W$ since, in free rotation, all positions are equally probable and the weight factor as a function of position reduces to unity. Integral (3) is the imaginary part of

$$
\frac{1}{\pi x} \int_{0}^{\pi} \frac{\exp \left[i x(1-p \cos \beta)^{\frac{1}{2}}\right]}{(1-p \cos \beta)^{\frac{1}{2}}} d \beta .
$$

This integral will now be evaluated by the saddle point method, the details of which have been clearly presented by Debye. ${ }^{2}$ The principle of this method is that integrals of the type in (4) can be evaluated asymptotically by considering the contribution in the vicinity of the extrema of $f(\beta)=(1-p \cos \beta)^{\frac{\pi}{2}}$, which occur when $\beta=0$ and $\beta=\pi$, the saddle points. The evaluation is accomplished by choosing a contour of integration along a path of steepest descent (steepest decrease of magnitude of the integrand) through the saddle points.

In order to obtain now the contribution from the neighborhood of $\beta=0$, we define a new variable,

$$
t=f(\beta)-f(0)=(1-p \cos \beta)^{\frac{1}{2}}-(1-p)^{\frac{1}{2}} .
$$

From (5) we get

$$
\begin{aligned}
\sin \beta(t) & =\frac{2(1-p)^{\frac{1}{2}}}{p^{\frac{1}{2}}} t^{\frac{1}{2}}\left(1-\frac{(2-3 p) t}{2 p(1-p)^{\frac{1}{2}}}\right. \\
& \left.-\frac{t^{2}}{p}-\frac{t^{3}}{4 p(1-p)^{\frac{1}{2}}}\right)^{\frac{1}{2}}, \\
& =\frac{2(1-p)^{\frac{1}{4}}}{p^{\frac{1}{2}}} t^{\frac{1}{2}} / \sum_{n=0}^{\infty} b_{n} t^{n} .
\end{aligned}
$$

The values of $b_{0}, b_{1}, b_{2}$, and $b_{3}$ are given along with the final expression.

We now use (5) and (6) to express integral (4) as a function of $t$, and then make an additional

\footnotetext{
${ }^{2}$ P. Debye, Math. Ann. 67, 535 (1909).
}

change of variable, setting $i x t=-\tau$. This gives

$$
\begin{aligned}
& I_{0} \sim \frac{\exp \left[i x(1-p)^{\frac{1}{2}}\right]}{\pi x(-i x)^{\frac{1}{2}}(1-p)^{\frac{1}{4}} p^{\frac{1}{2}}} \int_{0}^{\infty} \frac{e^{-\tau}}{\tau^{\frac{1}{2}}} \\
& \quad \times\left(1+\frac{i b_{1}}{x} \tau-\frac{b_{2}}{x^{2}} \tau^{2}-\frac{i b_{3}}{x^{3}} \tau^{3}+\cdots\right) d \tau .
\end{aligned}
$$

The choice of a contour of integration along the path of steepest descent through the saddle point allows us to choose $\infty$ as the upper limit. Since

$$
\int_{0}^{\infty} e^{-\tau} \tau^{\nu} d \tau=\mathrm{\Gamma}(\nu+1)
$$

we obtain

$$
\begin{aligned}
I_{0} & \sim \frac{\exp \left[i x(1-p)^{\frac{1}{2}}+i \pi / 4\right]}{\pi x(x p)^{\frac{1}{2}}(1-p)^{1}}[\Gamma(1 / 2) \\
& \left.+\frac{i b_{1}}{x} \Gamma(3 / 2)-\frac{b_{2}}{x^{2}} \Gamma(5 / 2)-\frac{i b_{3}}{x^{3}} \Gamma(7 / 2)+\cdots\right],
\end{aligned}
$$

by choosing $(-i)^{\frac{1}{2}}=e^{-i \pi / 4}$.

A similar term for the contribution from the neighborhood of $\beta=\pi$ can be obtained in the same way. By defining a new variable

$$
t_{1}=f(\beta)-f(\pi)=(1-p \cos \beta)^{\frac{1}{2}}-(1+p)^{\frac{1}{1}},
$$

it is then found that

$$
\begin{aligned}
\sin \beta\left(t_{1}\right)= & \frac{2(1+p)^{\frac{1}{4}}}{p^{\frac{1}{2}}} i t_{1^{\frac{1}{2}}}\left(1+\frac{(2+3 p) t_{1}}{2 p(1+p)^{\frac{1}{2}}}\right. \\
& \left.\quad+\frac{t_{1}{ }^{2}}{p}+\frac{t_{1^{3}}}{4 p(1+p)^{\frac{1}{2}}}\right)^{\frac{1}{2}}, \\
= & \frac{2(1+p)^{\frac{1}{4}}}{p^{\frac{1}{3}}} i t_{1^{\frac{1}{3}}} / \sum_{n=0}^{\infty} b_{n}{ }^{\prime} t_{1}{ }^{n} .
\end{aligned}
$$

We finally obtain

$$
\begin{aligned}
& I_{x} \sim \frac{\exp \left[i x(1+p)^{\frac{1}{2}}-i \pi / 4\right]}{\pi x(p x)^{\frac{1}{2}}(1+p)^{\frac{1}{2}}} \\
& \times\left[\Gamma(1 / 2)+\frac{i b_{1}{ }^{\prime}}{x} \Gamma(3 / 2)\right. \\
& \left.-\frac{b_{2}{ }^{\prime}}{x^{2}} \Gamma(5 / 2)-\frac{i b_{3}{ }^{\prime}}{x^{3}} \Gamma(7 / 2)+\cdots\right] .
\end{aligned}
$$


We now have the complete evaluation of the original integral (3) in terms of the contributions from the two saddle points. It is the imaginary part of

$$
\begin{aligned}
& \frac{\exp \left[i x(1-p)^{\frac{1}{3}}+i \pi / 4\right]}{x^{\mathrm{l}}(p \pi)^{\frac{1}{2}}(1-p)^{\frac{1}{2}}} \\
& \quad \times\left(1+\frac{i b_{1}}{2 x}-\frac{3 b_{2}^{\prime}}{4 x^{2}}-\frac{15 i b_{3}}{8 x^{3}}+\cdots\right) \\
& +\frac{\exp \left[i x(1+p)^{\frac{1}{2}}-i \pi / 4\right]}{x^{\frac{3}{2}}(p \pi)^{\frac{1}{2}}(1+p)^{\frac{1}{2}}} \\
& \quad \times\left(1+\frac{i b_{1}^{\prime}}{2 x}-\frac{3 b_{2}^{\prime}}{4 x^{2}}-\frac{15 i b_{3}^{\prime}}{8 x^{3}}+\cdots\right),
\end{aligned}
$$

and taking the imaginary part we have,

$$
\begin{aligned}
& I \sim \frac{1}{(\pi p)^{\frac{1}{2} x^{\frac{1}{2}}}} \\
& \quad \times\left[S(x, p, \pi / 4) \sigma_{1}(p)+C(x, p, \pi / 4) \sigma_{2}(p)\right. \\
& \quad+S(x,-p,-\pi / 4) \sigma_{1}(-p) \\
& \left.\quad+C(x,-p,-\pi / 4) \sigma_{2}(-p)\right],
\end{aligned}
$$$$
\text { where }
$$

$$
\begin{aligned}
S(x, p, \pi / 4) & =\frac{\sin \left[x(1-p)^{\frac{1}{2}}+\pi / 4\right]}{(1-p)^{\frac{1}{2}}}, \\
C(x, p, \pi / 4) & =\frac{\cos \left[x(1-p)^{\frac{1}{2}}+\pi / 4\right]}{(1-p)^{\frac{3}{4}}}, \\
\sigma_{1}(p) & =1-\frac{3 b_{2}}{4 x^{2}}+\cdots, \\
\sigma_{2}(p) & =\frac{b_{1}}{2 x}-\frac{15 b_{3}}{8 x^{3}}+\cdots,
\end{aligned}
$$

and

and

$$
b_{1}=\frac{2-3 p}{4 p(1-p)^{\frac{1}{3}}}, \quad b_{2}=\frac{12-20 p+11 p^{2}}{32 p^{2}(1-p)},
$$

$$
b_{3}=\frac{40-132 p+166 p^{2}-79 p^{3}}{128 p^{3}(1-p)^{1}} .
$$

The Debye formula is obtained if one expresses $x$ and $p$ in terms of $s, l_{2}$ and $l_{1}$, sets $\sigma_{1}=1$ and $\sigma_{2}=0$ in (13), and if the molecule considered has the dimensions such that $l_{2}{ }^{2}-l_{1}{ }^{2}=4 a^{2}$, where $a$ is the radius of rotation. (An example is the case of a molecule with an identical group rotating at each end of a bond.)

\section{DISCUSSION}

It can be shown that the series in (13) are asymptotic in the sense of Poincare. ${ }^{3}$ Thus these series are divergent and this property is manifested by an increase in magnitude of successive terms after a finite number of terms of steadily decreasing magnitude have been passed. In applying the series, the terms which are successively increasing are not included and the approximation will be improved by taking more and more of the successively decreasing terms. The magnitude of the error resulting from cutting the series off at a particular term is roughly of the order of magnitude of the next term in the series.

It is customary in using the visual method ${ }^{4}$ for studying electron diffraction patterns of gas molecules to calculate theoretical intensity curves from $s=2$ to $s=20$ or further and to attach quantitative significance at $s$ values greater than 5 . It is therefore desirable to evaluate the magnitude of successive terms in the asymptotic series in the vicinity of $s=5$ for some typical molecules. (It should be noted however that with improvement in methods for obtaining accurate diffraction intensities, it may be desirable to consider quantitatively the intensities at values of $s$ less than 5.)

In discussing the applicability of the derived series it is somewhat less cumbersome to refer to the complex expression (12). However, the remarks to be made are readily applied to the final form (13). Two different molecules were chosen for discussion. Hexamethylethane was chosen because a study had been made on this molecule by S. H. Bauer and J. Y. Beach ${ }^{5}$ using Debye's formula and it represents a very favorable case. The second example concerns the rotation of the 3 and $\mathbf{3}^{\prime}$ carbon atoms in biphenyl, and, as will be seen, is less favorable for the application of the Debye formula. In Table I, the magnitude of the second and third terms included under the parentheses of each of the two series in (12) is recorded for three different values of $s$. The calculations were based on a hexamethylethane

${ }^{3}$ E. T. Whittaker and G. N. Watson, Modern Analysis (The Macmillan Company, New York, 1943), American edition, p. 151.

${ }^{4}$ L. O. Brockway, Rev. Mod. Phys. 8, 231 (1936).

5 S. H. Bauer and J. Y. Beach, J. Am. Chem. Soc. 64, 1142 (1942). 
model in which the carbon to carbon distances were taken to be $1.54 \mathrm{~A}$ and all the angles were considered tetrahedral. In Table II are recorded the same mathematical quantities for the 3 and $3^{\prime}$ carbon atoms in a model of biphenyl which has

TABLE I. Evaluation of successive coefficients in the asymptotic series for a model of hexamethylethane.

\begin{tabular}{lllll}
\hline \hline & $\left|\frac{b_{1}}{2 x}\right|$ & $\left|\frac{3 b_{2}}{4 x^{2}}\right|$ & $\left|\frac{b_{1}}{2 x}\right|$ & $\left|\frac{3 b_{2}}{4 x^{2}}\right|$ \\
\hline$s=2$ & 0.05 & 0.03 & 0.13 & 0.06 \\
$s=5$ & 0.02 & 0.005 & 0.05 & 0.01 \\
$s=10$ & 0.01 & 0.001 & 0.03 & 0.002 \\
\hline \hline
\end{tabular}

regular benzene rings with a carbon to carbon distance in the ring taken as $1.39 \mathrm{~A}$ and a separation between the rings of $1.52 \mathrm{~A}$.

Whereas these two examples are by no means exhaustive, significant information may be obtained by examining the tables. The values for the terms included in the tables must be compared with unity, the leading term in the series, and thus the approximate magnitude of the error in omitting successive terms is readily obtained. For instance, for hexamethylethane the second term of the first series in expression (12) is only about 2 percent of the first term for $s=5$. The second term in the second series for $s=2$ is about 13 percent of the first but this is in a region which is insignificant so far as the visual method is concerned. However for biphenyl in Table II, we see that the third term is larger than the second for $s=2$ and so calculations would include only the second term introducing a rather large error. It is also seen that rather large errors occur in omitting successive terms in the region of $s=5$ to 10 .

\section{CONCLUSIONS}

Several conclusions can be drawn :

1. Consistent with the spirit of the saddle point method, the accuracy of the asymptotic evaluation increases as $s$ increases.

2. The asymptotic series may be used to estimate the error involved in using the Debye formula and affords a scheme for reducing the error by utilizing additional terms.

3. When the rotating atoms are far apart, the leading terms in the series may decrease in magnitude more slowly. This is illustrated in the case of biphenyl where the effect of the smaller value of $p$ as compared to the value in hexa-

TABLE II. Evaluation of successive coefficients in the asymptotic series for a model of biphenyl (re: $3,3^{\prime}$ carbon atoms).

\begin{tabular}{lllll}
\hline \hline & $\left|\frac{b_{1}}{2 x}\right|$ & $\left|\frac{3 b_{2}}{4 x^{2}}\right|$ & $\left|\frac{b_{1}{ }^{\prime}}{2 x}\right|$ & $\left|\frac{3 b_{2}{ }^{\prime}}{4 x^{2}}\right|$ \\
\hline$s=2$ & 0.23 & 0.28 & 0.28 & 0.32 \\
$s=5$ & 0.09 & 0.05 & 0.11 & 0.05 \\
$s=10$ & 0.05 & 0.01 & 0.06 & 0.01 \\
\hline \hline
\end{tabular}

methylethane more than compensates for the effect of the increase in the value of $x$ due to the larger separation. Since the barriers which restrict rotation are smaller when the rotating groups are farther apart, we can expect that the asymptotic series will find its greatest application in such cases.

\section{ACKNOWLEDGMENT}

I wish to express my appreciation to Professor Otto Laporte for his help in deriving the asymptotic series. 